\title{
Abordagem de Flutter Atrial Neonatal: Uma Série de Casos
}

\author{
Neonatal Atrial Flutter Approach: A Case Series \\ Fernanda Pessa Valente1, Gustavo Henrique Belarmino Góes ${ }^{2, *}$, Caroline Bernardi Fabro², \\ Afonso Luiz Tavares Albuquerque ${ }^{3}$, Dário Celestino Sobral Filho²
}

ORCID IDs

Valente FP (iD https://orcid.org/0000-0002-9713-6988

Góes GHB (D) https://orcid.org/0000-0003-3366-1182

\begin{abstract}
RESUMO
Objetivo: Este estudo teve como objetivo analisar as opções terapêuticas dos pacientes com flutter atrial (FLA) neonatal, considerando os métodos diagnósticos disponíveis e o prognóstico desses pacientes. Metodologia: Foi realizado um estudo retrospectivo através da revisão dos prontuários de uma série de sete pacientes com fibrilação atrial (FA) diagnosticada durante o período fetal ou neonatal. O tempo de seguimento desses pacientes variou de 7 meses a 3 anos e 8 meses (média: 1 ano). Os dados clínicos para o diagnóstico incluíram frequência cardíaca sustentada superior a 180 bpm, que foi confirmada em todos os pacientes por um eletrocardiograma de 12 derivações. Resultados: Quatro $(57,1 \%)$ dos sete pacientes estudados eram do sexo masculino. A maioria dos pacientes revelou arritmia cardíaca durante o período intrauterino, quando examinados por ultrassom fetal no terceiro trimestre de gestação (5 pacientes, ou seja 71,2\%). Apenas à mãe do Paciente 2 foi administrada digoxina antes do parto. A taxa atrial da taquiarritmia revelou uma média de 375 bpm, com um aumento de até 500 bpm. A condução atrioventricular apresentou uma relação de 2:1 em todos os pacientes, com variações de 3:1 e 4:1 observadas nos Pacientes 1, 3 e 6. A frequência ventricular variou de 188 a 250 bpm. Todos os pacientes revelaram características típicas e anti-horárias do eletrocardiograma. A cardioversão elétrica sincronizada foi o tratamento de escolha em 6 pacientes (85,7\%), com uma dose de $1 \mathrm{~J} / \mathrm{kg}$. Conclusão: Diagnóstico precoce, tratamento prévio e cardioversão elétrica sincronizada indicam um excelente prognóstico, e o tratamento de manutenção prolongada pode ser desnecessário.
\end{abstract}

PALAVRAS-CHAVE: Arritmia cardíaca; Supraventricular; Neonatal.

\author{
Albuquerque ALT (D) https://orcid.org/0000-0003-3801-9201 \\ Fabro CB (D) https://orcid.org/0000-0003-4522-8810 \\ Sobral Filho DC (D) https://orcid.org/0000-0002-5301-7741
}

\begin{abstract}
Objective: This study set out to analyze the therapeutic options of patients with neonatal atrial flutter (AFL), considering the diagnostic methods available and the prognosis of these patients. Methodology: A retrospective study was performed by reviewing the medical records of a series of seven patients with atrial fibrillation (AF) diagnosed during fetal or neonatal period. The follow-up time of these patients ranged from 7 months to 3 years and 8 months (mean: 1 year). The clinical data for the diagnosis included sustained heart rate greater than 180 bpm, which was confirmed in all patients by a 12-lead electrocardiogram. Results: Four (57.1\%) of the 7 patients studied were male. Most of the patients revealed cardiac arrhythmia during the intrauterine period when screened by fetal ultrasound in the third trimester of gestation (5 patients, i.e. 71.2\%). Only the mother of Patient 2 was administered digoxin before childbirth. The atrial rate of the tachyarrhythmia revealed a mean of 375 bpm, with an increase of up to $500 \mathrm{bpm}$. Atrioventricular conduction presented a 2:1 ratio in all patients, with variations of 3:1 and 4:1 observed in Patients 1, 3 and 6 . The ventricular rate ranged from 188 to $250 \mathrm{bpm}$. All patients revealed typical and counter-clockwise electrocardiogram characteristics. Synchronized electrical cardioversion was the treatment of choice in 6 patients (85.7\%), with a dose of $1 \mathrm{~J} / \mathrm{kg}$. Conclusion: Early diagnosis, prior treatment, and synchronized electrical cardioversion indicate an excellent prognosis, and prolonged maintenance treatment may be unnecessary.
\end{abstract}

KEYWORDS: Cardiac arrhythmia; Supraventricular; Neonatal.

1.Instituto de Medicina Integral Professor Fernando Figueira - Recife (PE), Brasil.

2.Universidade de Pernambuco - Faculdade de Ciências Médicas - Recife (PE), Brasil.

3.Pronto Socorro Cardiológico de Pernambuco - Recife (PE), Brasil.

Recebido: Jan, 132020 | Aceito: Jan. 27, 2020

*Autor correspondente: gustavogoesmt@gmail.com

Editor de seção: José Tarciso Medeiros de Vasconcelos 


\section{INTRODUÇÃO}

O flutter atrial (FLA) é definido como uma taquiarritmia supraventricular que se origina de um circuito macrorreentrante, na maioria das vezes no átrio direito, gerando atividade elétrica atrial contínua. O FLA pode ser típico, que é o tipo mais frequente, quando o istmo cavo-tricúspide faz parte do seu circuito; ou atípico, quando essa região anatômica é excluída. Vários autores também se referem a esta última como taquicardia atrial macrorreentrante. O FLA típico pode ser no sentido anti-horário quando a despolarização do septo atrial ocorre a partir do fundo, ou no sentido horário, na situação inversa. Os pacientes com risco aumentado de FLA incluem idosos e pessoas com doença cardíaca estrutural, insuficiência cardíaca ou doença pulmonar obstrutiva. A frequência atrial varia de 240 a $340 \mathrm{bpm}$ e a frequência ventricular depende do grau de bloqueio no nó atrioventricular, resultando em relações atrioventriculares variáveis, tais como 1:1, $2: 1$ (mais comum), 3:1, ou 4:1 .

O mecanismo arritmogênico em recém-nascidos é o mesmo. No entanto, como a maioria dos neonatos revela um coração estruturalmente normal, a etiologia não está bem definida. A taxa atrial apresenta valores mais altos em neonatos do que em adultos, com uma média de 440 bpm. Em crianças mais velhas, a taxa é cerca de 300 bpm. A relação atrioventricular (AV) também varia, sendo 2:1 a mais frequente ${ }^{2}$.

As arritmias cardíacas são encontradas em cerca de $2 \%$ dos fetos. Nessa população, eles são considerados um fator de risco e são uma das principais indicações para a ecocardiografia fetal. A suspeita de arritmia fetal inclui o seguinte: frequência cardíaca (FC) sustentada acima de $180 \mathrm{bpm}$, batimentos cardíacos irregulares repetitivos e hidropisia fetal inexplicada. Em todos os casos, a exclusão da lesão cardíaca subjacente é obrigatória ${ }^{3}$.

A ausência de estudos multicêntricos na população pediátrica indica falta de dados precisos relacionados à incidência, etiologia, risco de tromboembolismo e medidas terapêuticas baseadas em evidências para o tratamento da FLA $^{4}$.

\section{MÉTODOS}

Foi realizado um estudo retrospectivo através da revisão dos prontuários de uma série de 7 pacientes com FLA diagnosticado durante o período fetal ou neonatal. O período de seguimento desses pacientes variou de 7 meses a 3 anos e 8 meses (média: 1 ano). Os pacientes nasceram em hospitais de referência para neonatologia na cidade do Recife, Brasil, e foram transferidos para os centros de referência em cardiologia pediátrica após o diagnóstico de taquiarritmia. Os dados clínicos para diagnóstico incluíram frequência cardíaca sustentada superior a 180 bpm. O diagnóstico de FLA foi confirmado em todos os pacientes por meio de um eletrocardiograma (ECG) de 12 derivações.

\section{RESULTADOS}

Quatro $(57,1 \%)$ dos sete pacientes estudados eram do sexo masculino (Tabela 1). A maioria deles revelou arritmia cardíaca durante o período intrauterino, quando rastreados por ultrassom fetal no terceiro trimestre de gestação (5 pacientes, ou seja 71,2\%). Mesmo quando uma arritmia intrauterina era suscetível, os ecocardiogramas fetais não eram realizados devido à idade gestacional avançada. Durante a gestação, foram observadas as seguintes complicações: diabetes gestacional, pielonefrite, polidrâmnio e prematuridade às 36 semanas (Paciente 4), infecção do trato urinário próximo ao parto e prematuridade às 32 semanas (Paciente 5), alcoolismo e tabagismo às 39 semanas (Paciente 7). Apenas à Paciente 2 foi administrada digoxina $(0,25 \mathrm{mg} / \mathrm{dia})$ antes do parto.

Ao nascimento, apenas o Paciente 5 foi considerado como tendo hipóxia leve, de acordo com o escore de Apgar, e penicilina cristalina e gentamicina foram administradas via intravenosa durante 7 dias. Os sinais de insuficiência cardíaca estavam ausentes nos pacientes ao nascimento ou no momento do diagnóstico de arritmia. O ecocardiograma transtorácico foi realizado em todos os pacientes, sendo a comunicação interatrial (CIA) a cardiopatia congênita mais frequente (4 pacientes, ou seja, $57,1 \%$ ), com repercussão hemodinâmica ocorrendo apenas no Paciente 7 . Todos os pacientes revelaram função ventricular normal (Tabela 2).

Considerando as características eletrocardiográficas, a taxa atrial da taquiarritmia revelou uma média de $375 \mathrm{bpm}$, com um aumento de até 500 bpm (Fig. 1). A condução atrioventricular apresentou uma relação de 2:1 em todos os pacientes, com variações de 3:1 e 4:1 observadas nos Pacientes 1, 3 e 6. A frequência ventricular variou de 188 a $250 \mathrm{bpm}$. Todos os pacientes revelaram características típicas e anti-horárias do ECG. 
Tabela 1. Características clínicas dos pacientes com flutter atrial.

\begin{tabular}{|c|c|c|c|c|}
\hline Pacientes & $\begin{array}{l}\text { Idade no diagnóstico } \\
\text { (MSC) }\end{array}$ & Sexo & Complicações neonatais & $\begin{array}{c}\text { Insuficiência } \\
\text { cardíaca }\end{array}$ \\
\hline 1 & 37 semanas & Feminino & Não & Não \\
\hline 2 & 37,2 semanas & Masculino & Não & Não \\
\hline 3 & 37,3 semanas & Masculino & Não & Não \\
\hline 4 & 8 h de vida (36 semanas) & Feminino & DMG + pielonefrite + polidrâmnios + prematuridade & Não \\
\hline 5 & 32 semanas & Masculino & ITU tratada + prematuridade + baixo peso ao nascer & Não \\
\hline 6 & 6 dias & Feminino & Não & Não \\
\hline 7 & 72 h de vida (39,2 semanas) & Masculino & Consumo de álcool + tabagismo + PIG & Não \\
\hline
\end{tabular}

MSC: Método somático de Capurro; DMG: diabetes melito gestacional; ITU: infecção do trato urinário; PIG: pequeno para a idade gestacional.

Tabela 2. Cardiopatia congênita associada a flutter atrial e diagnosticada por ecocardiograma transtorácico.

\begin{tabular}{ccc}
\hline Pacientes & Cardiopatia congênita & Sexo \\
\hline 1 & FOP & Feminino \\
2 & FOP + ampliação do AD & Masculino \\
$\mathbf{3}$ & CIA tipo ostium secundum & Masculino \\
$\mathbf{4}$ & CIA tipo ostium secundum & Feminino \\
$\mathbf{5}$ & ASA + CIA pequena tipo ostium secundum + pequena PCA & Masculino \\
$\mathbf{6}$ & FOP & Feminino \\
$\mathbf{7}$ & CIA tipo ostium secundum + AD e ampliação VD & Masculino \\
\hline
\end{tabular}

FOP: forame oval patente; AD: átrio direito; CIA: comunicação interatrial; ASA: aneurisma do septo atrial; PCA: persistência do canal arterial; VD: ventrículo direito.

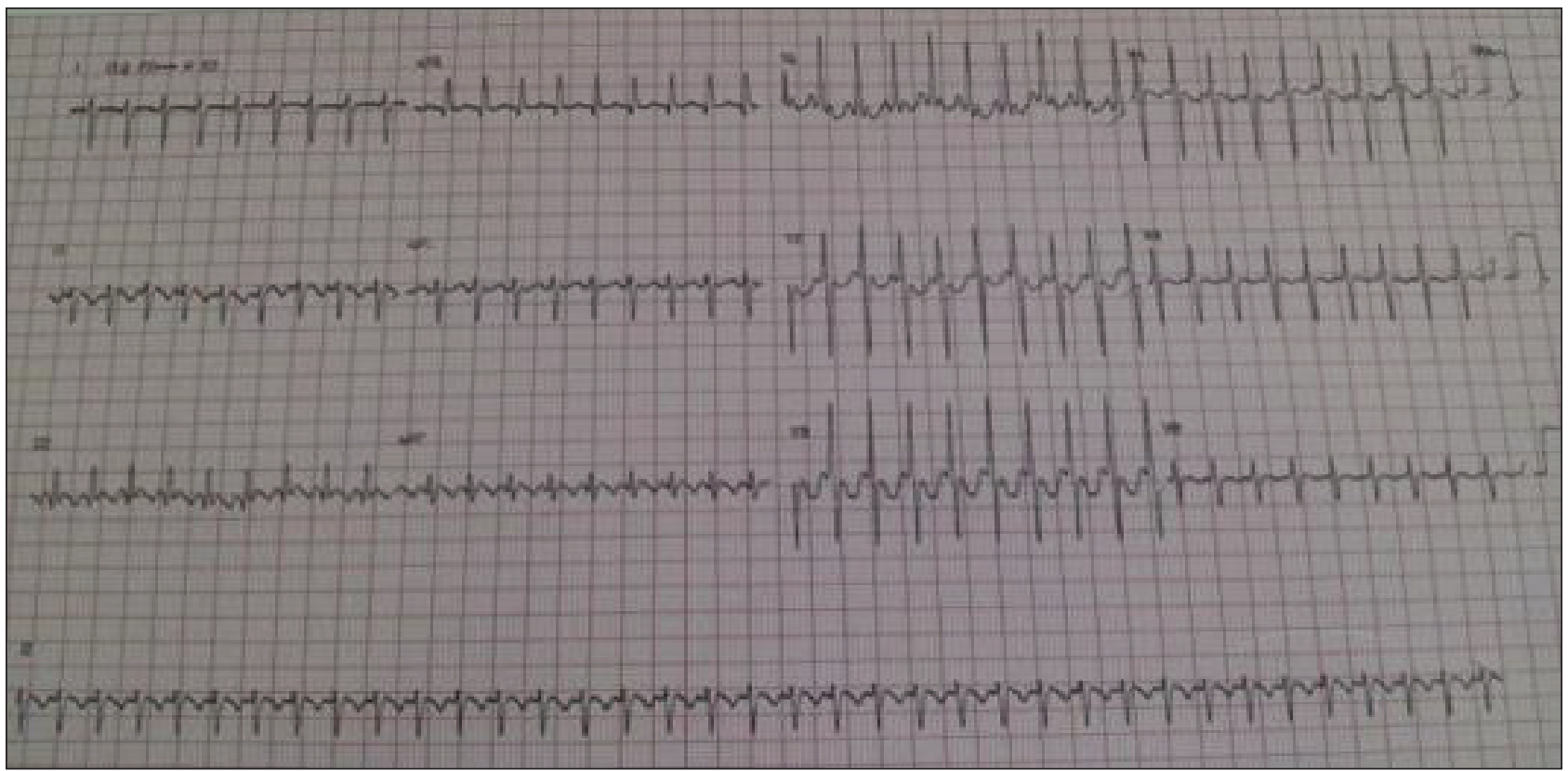

Figura 1. Eletrocardiograma mostrando flutter atrial com frequência atrial de aproximadamente 500 bpm, relação atrioventricular 2:1 e frequência ventricular de cerca de 250 bpm (Paciente 4).

A cardioversão elétrica sincronizada (CVES) foi o tratamento de escolha em 6 pacientes $(85,7 \%)$, com uma dose de $1 \mathrm{~J} / \mathrm{kg}$. Foi realizada nas primeiras 24 h após o nascimento em 3 pacientes (Pacientes 1, 2 e 4). Paciente 3 recebeu CVES 48 h após o nascimento; Paciente 6 no $6^{\circ}$ dia após o nascimento; e Paciente 7 em 72 h após o nascimento. Apenas o Paciente 4 apresentou depressão respiratória durante o procedimento, requerendo intubação orotraqueal e reaplicação do CVES, com a mesma dose da primeira, desta vez com resultado positivo. O paciente foi extubado 
em 24 h sem maiores complicações. Após o CVES, todos os pacientes receberam amiodarona intravenosa na dose de $10 \mathrm{mg} / \mathrm{kg} / \mathrm{dia}$, e a dosagem foi reduzida para $5 \mathrm{mg} / \mathrm{kg} / \mathrm{dia}$ por via oral após $48 \mathrm{~h}$. Apenas o Paciente 5 foi submetido a tratamento farmacológico: inicialmente com adenosina ( 2 doses de $0,1 \mathrm{mg} / \mathrm{kg}$ cada); $(0,01 \mathrm{mg} / \mathrm{kg} / \mathrm{dia}) ;$ e posteriormente com amiodarona (dose EV $10 \mathrm{mg} / \mathrm{kg}$ e manutenção EV $5 \mathrm{mg} / \mathrm{kg} / \mathrm{dia}$ ). No terceiro dia após o nascimento, usando amiodarona, o ritmo sinusal foi invertido. Apenas o Paciente 1 apresentou recidiva de taquiarritmia após $2 \mathrm{~h}$ de CVES. Durante esse período, a dose de amiodarona foi aumentada para $15 \mathrm{mg} / \mathrm{kg} / \mathrm{dia}$ por via intravenosa e um novo CVES foi programado; entretanto, houve uma reversão para o ritmo sinusal após 14 $\mathrm{h}$ sem a necessidade de cardioversão. A terapia anticoagulante não foi realizada antes do CVES em nenhum dos pacientes.

Três pacientes $(42,9 \%)$ apresentaram um intervalo PR prolongado no ECG após a cardioversão (Tabela 3). Não foram observadas arritmias adicionais após a restauração do ritmo sinusal. Todos os pacientes tiveram alta em tratamento farmacológico com amiodarona (dose $5 \mathrm{mg} / \mathrm{kg} / \mathrm{dia}$ ), com internação hospitalar média de 7 dias, variando de 3 a 12 dias. O tratamento de manutenção com amiodarona variou de 1 a 6 meses, com uma média de 3 meses. Devido à curta duração do tratamento, não foram realizados testes de função tireoidiana. $\mathrm{O}$ teste de mancha de sangue também não apresentou alterações. Atualmente, todos os pacientes são acompanhados como pacientes ambulatoriais, assintomáticos, com desenvolvimento neuropsicomotor adequado e sem novos episódios de FLA.

Tabela 3. Mudanças eletrocardiográficas após cardioversão elétrica sincronizada.

\begin{tabular}{cc}
\hline Pacientes & Mudanças após CVES \\
\hline 1 & Não \\
2 & Ampliação PRi (130 ms) \\
3 & Ampliação PRi (120 ms) \\
4 & Não \\
5 & Ampliação PRi (140 ms) \\
6 & Não \\
$\mathbf{7}$ & Não \\
\hline
\end{tabular}

CVES: cardioversão elétrica sincronizada; PRi: intervalo PR.

\section{DISCUSSÃO}

Atualmente, o FLA pode ser agrupado em duas situações clínicas diferentes na população pediátrica: no período fetal, neonatal e infantil, geralmente com um coração estruturalmente normal; e em crianças maiores com doença cardíaca estrutural associada (com ou sem cirurgia cardíaca) $)^{2,5}$.

Considerando a hipótese de arritmia cardíaca no feto, a idade gestacional ideal para a ecocardiografia fetal é provavelmente entre 16 e 22 semanas, já que a anatomia intracardíaca também deve ser avaliada para excluir a presença de cardiopatia congênita ${ }^{3,6}$, o que é praticamente inexistente, como demonstrado pelos pacientes que foram avaliados predominantemente a partir da 36a semana de gestação.

Uma vez confirmada a taquiarritmia fetal, os fatores que determinam o curso do tratamento in utero são a idade gestacional, doença cardíaca congênita associada, sintomas de insuficiência cardíaca e, particularmente, a presença de hidropisia e a duração da taquicardia ${ }^{7}$. Simpson et al. relataram que $65 \%$ das taquicardias em fetos eram persistentes, e a incidência de hidropisia foi de $41 \%{ }^{8}$. Apenas à mãe de um paciente deste estudo foi administrada digoxina $(0,25 \mathrm{mg} /$ dia $)$ antes do parto, uma droga de primeira linha para fetos não hidrofóbicos, como relatado por Batra et al. ${ }^{9}$. Em fetos, a flecainida é a droga mais indicada ${ }^{9}$ Lisowski et al. ${ }^{10}$, em estudo retrospectivo com 45 pacientes com ABI perinatal (44 com diagnóstico fetal e 1 imediatamente após o nascimento), avaliaram a eficácia do tratamento intrauterino. Eles concluíram que a digoxina não conseguiu prevenir a recorrência do FLA em um quarto dos pacientes, enquanto não foram observadas recidivas no grupo do sotalol. Os pacientes em que a arritmia recidivou ao nascimento foram tratados com CVES, sem recidivas posteriores. Nos casos de arritmia persistente, houve complicações como hidropisia e comprometimento neurológico, enfatizando a importância do tratamento precoce dessa arritmia em relação ao prognóstico dos pacientes; entretanto, nos casos que reverteram ao ritmo sinusal, a terapia medicamentosa de manutenção foi desnecessária.

Flutter atrial em fetos e recém-nascidos é, em geral, clinicamente bem tolerado ${ }^{8}$. A taxa atrial nesses pacientes apresenta valores mais altos do que em adultos, com média de $440 \mathrm{bpm}$, ou até $500 \mathrm{bpm}^{2,8}$. Os pacientes do presente estudo revelaram uma frequência cardíaca variando de 188 a $250 \mathrm{bpm}$, com média de 375 bpm, 
próxima à descrita na literatura, embora em um dos pacientes ela tenha aumentado para $500 \mathrm{bpm}$.

Pacientes com essa condição são geralmente assintomáticos, e se a relação $\mathrm{AV}$ apresentar um alto grau de bloqueio, a taxa ventricular estará próxima dos valores normais para a faixa etária em questão. Alternativamente, se a relação $A V$ for $1: 1$, pode ocorrer comprometimento cardiovascular e até causar mortalidade ${ }^{8}$. A relação AV mais comum é de 2:1 (75\% dos casos); consequentemente, a taxa ventricular varia de 150 a $250 \mathrm{bpm}$ na maioria dos pacientes ${ }^{4}$. Além disso, todos os pacientes revelaram uma frequência maior da razão $2: 1$, embora três pacientes tenham tido episódios da razão 3:1 e 4:1. De acordo com a literatura, a taxa ventricular dos pacientes do nosso estudo variou de 188 a $250 \mathrm{bpm}$.

Os defeitos cardíacos congênitos mais frequentes associados ao FLA são defeitos do septo atrial, aneurisma do septo atrial e doença de Ebstein ${ }^{5}$. Resultados semelhantes foram observados no presente estudo, sendo os defeitos mais frequentes o forame oval patente e CIA tipo ostium secundum, cada um deles presente separadamente em quatro pacientes. No entanto, em 1985, Garson Jr. et al. ${ }^{11}$ realizaram um estudo multicêntrico em Houston, Texas, compreendendo 380 pacientes diagnosticados com FLA, com idades entre 1 e 25 anos, dos quais apenas $8 \%$ revelaram doença cardíaca estrutural, mais frequentemente por transposição das grandes artérias, corações univentriculares, defeitos do septo interatrial, estenose pulmonar e tetralogia de Fallot, sendo a dilatação atrial o principal fator de risco associado ao FLA. As discrepâncias entre os resultados podem ser atribuídas ao fato de os autores terem incluído vários pacientes adultos, dos quais a fisiopatologia do FLA é aparentemente diferente dos casos congênitos ou neonatais.

Embora a terapia de primeira linha para FLA fosse farmacológica (digital, amiodarona e quinidina) até os anos 1990,3,4,12-15, o CVES é atualmente considerado o padrão ouro de tratamento ${ }^{4,16}$. Essa mudança foi refletida neste estudo, no qual $85,7 \%$ dos pacientes foram submetidos ao CVES com $1 \mathrm{~J} / \mathrm{kg}$, seguido de amiodarona intravenosa $(10 \mathrm{mg} / \mathrm{kg} / \mathrm{dia})$. Apenas um paciente foi tratado com medicação: inicialmente adenosina, seguido de C-delayside, e posteriormente amiodarona. Alguns autores também recomendam que, se a função ventricular for normal e o paciente estiver assintomático, o CVES pode ser retardado por $24 \mathrm{~h}$, já que $25 \%$ do FLA pode reverter espontaneamente ${ }^{4}$. Texter et al. ${ }^{16}$ estudaram uma revisão com 50 pacientes com idade inferior a 1 ano, diagnosticados com FLA ao nascimento e sem cirurgia cardíaca prévia, seguida no Texas Children's Hospital por um período de 25 anos. Concluíram que o diagnóstico de FLA ocorreu mais frequentemente nos dois primeiros dias após o nascimento e que não foi observada associação com cardiopatia congênita. A cardioversão elétrica sincronizada foi o tratamento mais eficaz para a obtenção do ritmo sinusal. Eles também estabeleceram que a FLA crônica tem um risco potencial de comprometimento cardiovascular, uma vez que os sinais de insuficiência cardíaca estariam relacionados com a duração prolongada da taquiarritmia. $\mathrm{Na}$ ausência de doença cardíaca estrutural, se tratada precocemente, o FLA tem baixo risco de recidiva, com excelente prognóstico e sem necessidade de terapia antiarrítmica crônica.

De acordo com as Diretrizes Brasileiras para Arritmias Cardíacas em Crianças e Cardiopatias Congênitas ${ }^{17}$, o FLA corresponde a aproximadamente $30 \%$ das taquiarritmias fetais. Além disso, o tratamento intrauterino depende da apresentação de taquiarritmia (sustentada ou não) e sua repercussão hemodinâmica, com graus variáveis de insuficiência cardíaca, disfunção ventricular e até mesmo hidropisia fetal. Imagens intermitentes e clinicamente ininterruptas podem ser mantidas com o acompanhamento clínico rigoroso do feto. Ocasionalmente, o uso de medicamentos antiarrítmicos maternos é indicado. Quando usado em combinação com a via auxiliar, o sotalol revelou uma taxa de $50-80 \%$ de reversão para um ritmo sinusal, sendo assim considerado como uma droga segura; no entanto o paciente deve permanecer internado, com monitoramento do intervalo QT devido aos fármacos efeito pró-rítmico. Digoxina e amiodarona são as outras opções possíveis, a primeira para aumentar o grau de bloqueio AV, diminuindo assim a resposta ventricular. A antecipação do nascimento dos filhos deve ser considerada se a mãe já estiver próxima do termo (avaliação multiprofissional). Após o parto, o tratamento deve ser individualizado, tendo o CVES como a melhor opção terapêutica.

Parte dos dados da série de casos em estudo correspondeu aos dados encontrados na literatura; entretanto, devido ao pequeno número de casos, não foi possível identificar os fatores de risco comuns associados à arritmia. Todos os 
casos foram classificados como FLA congênito, confirmado por ECG de 12 derivações. A cardiopatia mais frequente foi a comunicação interatrial ostium secundum; entretanto esse achado não foi considerado fator de risco para taquiarritmia, pois a repercussão hemodinâmica foi encontrada em apenas um paciente, enquanto em outro o ecocardiograma confirmou que não havia cardiopatia estrutural associada. $\mathrm{O}$ tratamento de escolha para a reversão ao ritmo sinusal foi o CVES, utilizado em seis pacientes. Esse método provou ser seguro e eficaz, uma vez que apenas um paciente teve uma depressão respiratória transitória. Houve apenas uma recorrência, mas que reverteu ao ritmo sinusal com o uso de amiodarona. Não ocorreram mais arritmias e os pacientes apresentaram boa evolução ao longo do acompanhamento clínico.

Flutter atrial continua sendo uma arritmia incomum na população pediátrica, com formas clínicas distintas, dependendo da faixa etária. O seu curso clínico, modo de apresentação, abordagem terapêutica e prognóstico são diferentes dos de crianças mais velhas e adultos.

$\mathrm{Na}$ vida intrauterina, o diagnóstico ainda é feito por meio do ecocardiograma fetal, e a confirmação após o nascimento é feita por ECG de 12 derivações. O diagnóstico é geralmente feito no terceiro trimestre de gestação e é mais frequente em pacientes do sexo masculino. O FLA geralmente apresenta uma progressão benigna, sem ocorrência de insuficiência cardíaca ou hidropisia, sendo o tratamento preparatório a exceção.

Em geral, a taxa atrial de taquiarritmia não excede 500 bpm, e a taxa de condução atrioventricular mais prevalente é de 2:1. A frequência ventricular atinge um máximo de $250 \mathrm{bpm}$, e o intervalo $\mathrm{PR}$ pode ser prolongado em até $50 \%$ dos casos. A cardioversão eléctrica sincronizada é o tratamento preferido, uma vez que é um método seguro e eficaz.

O presente estudo tem certas limitações, pois é retrospectivo e observacional. Desde o início, a pesquisa clínica e diagnóstica foi realizada exclusivamente pelo mesmo grupo de investigadores, reduzindo assim qualquer possível enviesamento.

Futuros estudos multicêntricos são necessários para melhor identificar a etiologia, incidência e fatores de risco para flutter, além de promover medidas terapêuticas baseadas em evidências nesses pacientes.

\section{CONCLUSÃO}

Diagnóstico, tratamento precoce e cardioversão elétrica sincronizada são propícios a um excelente prognóstico, e o tratamento de manutenção a longo prazo pode ser desnecessário.

\section{REFERÊNCIAS}

1. Issa ZF, Miller JM, Zipes DP. Typical atrial flutter. In: Clinical arrhythmology and electrophysiology. A companion to Braunwald's Heart Disease. Philadelphia: Elsevier/Saunders; 2012. p. 239-259.

2. Wren C. Atrial Flutter. In: Concise guide to pediatric arrhythmias. 1st ed. Oxford: Wiley-Blackwell; 2011. p. 40-42.

3. Gulletta S, Rovelli R, Fiori R, Bella PD. Multiple external electrical cardioversions for refractory neonatal atrial flutter. Pediatr Cardiol, 2012;33(2):354-6. https://doi. org/10.1007/s00246-011-0131-5

4. Abadir S, Fournier A, Dubuc M, Khairy P. Atrial flutter and fibrillation in the young patient without congenital heart disease. Progr Pediatr Cardiol, 2013; 35(1):41-8. https:// doi.org/10.1016/j.ppedcard.2012.11.006

5. Crochelet AS, Jacquemart C, Massin M. Repeated electrical cardioversions and amiodarone for recurrent neonatal atrial flutter. Arch Pediatr, 2015;22(10):1032-4. https://doi.org/10.1016/j.arcped.2015.06.006

6. Jone PN, Schowengerdt Jr KO. Prenatal diagnosis of congenital heart disease. Pediatr Clin N Am, 2009;56:(3)70915. https://doi.org/10.1016/j.pcl.2009.04.002

7. Jaeggi E, Öhman A. Fetal and neonatal arrhythmias. Clin Perinatol, 2016;43(1):99-112. https://doi.org/10.1016/j. clp.2015.11.007

8. Woo J, Khan O, Caldarelli L, Williams P. Tachycardia in the neonate. Pediatr Ann, 2015;44(10):e247-50. https://doi. org/10.3928/00904481-20151012-09

9. Pike JI, Krishnan A, Kaltman J, Donofrio MT. Fetal and neonatal atrial arrhythmias: an association with maternal diabetes and neonatal macrosomia. Prenat Diagn, 2013;33(12):1152-7. https://doi.org/10.1002/pd.4210 
10. Kwok SY, Davis AM, Hutchinson D, Pflaumer A. Successful ablation of refractory neonatal atrial flutter. Heart Rhythm Case Rep, 2015;1(4):245-8. https://doi. org/10.1016/j.hrcr.2015.03.010

11. Garson Jr A, Bink-Boelkens M, Hesslein PS, Hordof AJ, Keane JF, Neches WH, Porter CJ. Atrial flutter in the young: a collaborative study of 380 cases. J Am Coll Cardiol, 1985;6(4):871-8. https://doi.org/10.1016/ s0735-1097(85)80497-6

12. Wacker-Gussmann A, Strasburger JF, Srinivasan S, Cuneo BF, LutterW, Wakai RT. Fetal atrial flutter: electrophysiology and associations with rhythms involving an accessory pathway. J Am Heart Assoc, 2016;5(6):e003673. https:// doi.org/10.1161/JAHA.116.003673

13. Moller JH, Davachi F, Anderson RC. Atrial flutter in infancy. J Pediatr, 1969;75(4):643-51. https://doi.org/10.1016/ s0022-3476(69)80461-0
14. Rowland TW, Mathew R, Chameides L, Keane JF. Idiopathic atrial flutter in infancy: a review of eight cases. Pediatrics, 1978;61(1):52-6.

15. Casey FA, McCrindle BW, Hamilton RM, Gow RM. Neonatal atrial flutter: significant early morbidity and excellent long-term prognosis. Am Heart J, 1997;133(3):302-6. https://doi.org/10.1016/s0002-8703(97)70224-2

16. Texter KM, Kertesz NJ, Friedman RA, Fenrich Jr AL. Atrial flutter in infants. J Am Coll Cardiol, 2006;48(5):1040-6. https://doi.org/10.1016/j.jacc.2006.04.091

17. Magalhães LP, Guimarães ICB, Melo SL, Mateo EIP, Andalaft RB, Xavier LFR, et al. Diretriz de arritmias cardíacas em crianças e cardiopatias congênitas SOBRAC e DCC - CP. Arq Bras Cardiol. 2016;107(1Supl.3):1-58. 